\title{
Determination of multiple vitamins in 178 patients undergoing chemotherapy for lung cancer
}

Lanqing Liang, Wei Yu, Boning Cai, Qianqian Wang, Xiang Huang, Jing Chen, Mingyue Zeng, Yao Wang, Baolin Qu, Fang Liu

Department of Radiotherapy, Chinese PLA General Hospital, Beijing, China

Submitted: 25 August 2020

Accepted: 8 November 2020

Arch Med Sci

DOI: $10.5114 /$ aoms/130302

Copyright @ 2020 Termedia \& Banach

\section{Abstract}

Introduction: The aim of the study was to investigate the changes in serum concentrations of nine vitamins in patients undergoing chemotherapy for lung cancer and explore their clinical values and influencing factors.

Material and methods: Patients receiving chemotherapy for lung cancer in our centre from February 2018 to May 2020 were enrolled in this study. Serum concentrations of the nine vitamins including vitamins $A, D, E, B_{9}$, $B_{12}, B_{1}, C, B_{2}$, and $B_{6}$ were measured in all subjects, and the changes in the concentrations of these vitamins were compared before and after 2 cycles of chemotherapy. In addition, the potential correlations of serum vitamin levels with age, gender, pathological type, and disease status were analysed. Results: In the 178 patients with lung cancer, there were different degrees of vitamin $A$, vitamin $D$, vitamin $C$, and in particular, vitamin $B$ deficiencies. Before chemotherapy, the concentrations of vitamin $A$ and vitamin $C$ were significantly different between males and females and among patients in different clinical stages (both $p<0.05$ ), the concentrations of vitamin $C$ and vitamin $B_{2}$ significantly differed among different pathological types of lung cancer $(p<0.05)$, and vitamin D level was significantly related to the disease status $(p<0.05)$. In addition, the proportion of vitamin $B_{2}$ deficiency differed significantly among different pathological types $(p<0.05)$. There were significant differences in the concentrations of vitamins $D, C$, and $B_{2}$ before and after chemotherapy (all $p<0.05$ ). There was a correlation between the change of serum vitamin $B_{1}$ concentration before and after chemotherapy and the change of body mass index $(p<0.05)$.

Conclusions: During chemotherapy, lung cancer patients are more likely to develop vitamins $A, D, C$, and $B_{2}$ deficiencies. Different vitamin deficiencies are related to gender, clinical stage, pathological type, and disease status. Vitamin determination and reasonable supplementation of nutrients in patients undergoing chemotherapy for lung cancer can help improve the nutritional status and increase chemotherapy tolerance.

Key words: lung cancer, vitamins, nutritional status, body mass index, chemotherapy.

\section{Introduction}

Lung cancer is the most prevalent and fatal malignancy worldwide. Its occurrence, development, and clinical prognosis are related to a variety of factors including smoking, environment, air pollution, diet, and gene expression [1-6]. Many studies have confirmed that, biologically, cancer is a metabolic disease $[7,8]$, and thus nutrition- and metabolism-regulating

\author{
Corresponding authors: \\ Fang Liu MD \\ Department of Radiotherapy \\ Chinese PLA \\ General Hospital \\ Beijing, China \\ E-mail: liufangfsq@163.com \\ Baolin Qu MD \\ Department of Radiotherapy \\ Chinese PLA \\ General Hospital \\ Beijing, China \\ E-mail: Qubl6212@sina.com
}


therapies have become new treatment strategies for tumours. The three major nutrients including carbohydrates, amino acids, and fats play key roles in regulating tumour metabolism [9], and the values of vitamins in the prevention and treatment of tumours have increasingly been recognised [10]. Nutritional microenvironment has become a hot research topic in recent years. Clinically, nutritional intervention and supportive treatment have become the core measures in the multidisciplinary treatment of tumours, and vitamins are an important component of parenteral nutrition supplementation for tumour patients. Therefore, monitoring the vitamin concentrations is essential for the nutritional supplementation of tumour patients. Malnutrition is common in patients with malignant tumours. Research has found that the incidence of malnutrition is $21.4-79.4 \%$ in tumour patients; in particular, the incidence of moderate to severe malnutrition ranges from $52.8 \%$ to $58.0 \%$, among which only about $30 \%$ of patients have received nutrition therapy $[11,12]$. Thus, the roles of nutritional assessment and nutritional intervention in cancer patients need to be further emphasised. The risk of malnutrition in patients with respiratory tumours is second only to those of head and neck tumours and gastrointestinal tumours. During chemotherapy, the side effects of drugs often lead to increased energy consumption, decreased dietary intake, and imbalanced food structure, which can easily cause malnutrition and vitamin deficiencies, resulting in decreased chemotherapy tolerance, prolonged hospital stay, lower quality of life, and higher medical expenses; some patients may even die of malnutrition [13]. Patients undergoing chemotherapy for lung cancer often suffer from bone marrow suppression, gastrointestinal reactions, electrolyte disturbances, and hypoproteinaemia, which further aggravate malnutrition. According to the ESPEN guidelines [14], nutrition treatment for cancer patients includes dietary guidance, nutrition education, nutrition risk screening, nutrition assessment, and nutrition intervention. Standardised nutrition therapy should follow the five-ladder treatment principle, which includes nutrition education, oral nutritional supplements (ONS), total enteral nutrition (EN), partial parenteral nutrition (PPN), and total parenteral nutrition (TPN). When the lower ladder cannot meet $60 \%$ of the target energy requirement within 3 to 5 days, the upper ladder will be selected. In addition to supplementing the three macronutrients - carbohydrate, fat, and protein, nutrition therapy for malnutrition should also meet micronutrient needs.

Micronutrients include vitamins and minerals. Although they are required in small amounts and generally cannot be synthesised by the human body, they are essential nutrients for the maintenance of human health and regulation of metabolism. Recent studies have found that vitamins A, C, D, E, and others participate in the pathogenesis of lung cancer, gastric cancer, colorectal cancer, and other tumours and affect clinical outcomes [15-17]. Nutrient supplementation is an important part of the multidisciplinary treatment of tumours. However, the use of vitamins in cancer patients is diverse. Multivitamin supplementation is usually based on experience or conventional therapeutic doses, and there is no clear theoretical evidence. A study in over 900 hospitalised patients with malignant tumours showed that vitamin $B_{1}$ decreased in patients with gastrointestinal tumours, vitamins $A$ and $B_{6}$ decreased in patients with urinary and reproductive system tumours, and vitamin C and D decreased in patients with lymphatic system tumours [18]. Hoffern et al. [19] determined vitamin levels in 91 patients with advanced lung cancer and found that vitamin D deficiency and vitamin $C$ deficiency accounted for $85 \%$ and $41 \%$, respectively. The degrees of vitamin deficiencies had certain correlations with the clinical discomforts. Vitamin D supplementation for 21 consecutive days significantly increased the plasma vitamin $D$ level in most patients but failed to improve negative emotions and alleviate symptoms.

In recent years, we have carried out vitamin testing for cancer inpatients and found that the nutritional status of vitamins differed among patients with different types of tumours. Recent studies on lung cancer and vitamins have been mainly focused on the role of a specific nutrient in tumour prevention and treatment, and few reports have described the monitoring and evaluation of multiple nutrients during chemotherapy for lung cancer patients. We investigated the changes in the concentrations of 9 vitamins before and after 2 cycles of chemotherapy in lung cancer patients and in different pathological types, and analysed the effects of chemotherapy drugs on vitamin levels. This study aimed to provide evidence of vitamin supplementation and supportive treatment for lung cancer patients, which may facilitate the targeted nutritional interventions, lower the incidence of malnutrition, and increase the tolerance and efficacy of chemotherapy in patients undergoing chemotherapy for lung cancer.

\section{Material and methods}

\section{General data}

A total of 178 patients who were admitted to the Department of Radiotherapy, Chinese PLA General Hospital were enrolled in this study. There were 138 males and 40 females, aged $26-88$ years $(60.13 \pm 9.47$ years). There were 74 cases of small cell lung cancer (SCLC) and 104 cases of non-small- 
cell lung cancer (NSCLC), and the treatment-naive patients accounted for $82.02 \%$ (Table I).

The inclusion criteria were as follows: a) with cytologically or histologically confirmed lung malignant tumours requiring chemotherapy; b) aged $\geq 18$ years; $c$ ) with a Karnofsky performance status (KPS) score $\geq 70$; and d) with an expected survival of $>6$ months.

The exclusion criteria included: a) with accompanying malignant tumours; b) with chemotherapy contraindications; c) undergoing immunotherapy or targeted therapy during chemotherapy; d) undergoing concurrent radiotherapy; e) with psychiatric disorders or severe psychological disease; f) failed to complete 2 cycles of chemotherapy; and g) having participated in clinical trials.

\section{Methods}

\section{Determination of vitamin concentrations}

Fasting venous blood samples $(4 \mathrm{ml})$ were collected from all patients. The concentrations of 9 vitamins were detected by the electrochemiluminescence method with an LK3000VI vitamin detector (Tianjin Lanbiao Electronic Technology Development Co., Ltd., Tianjin, China) in these lung cancer patients before chemotherapy and after 2 cycles of chemotherapy. The normal reference values of these vitamins used were as follows: vita$\min$ A, 0.52-2.2 $\mu \mathrm{mol} / \mathrm{l}$; vitamin D, 25-200 nmol/l; vitamin $\mathrm{E}, 10-15 \mu \mathrm{g} / \mathrm{ml}$; vitamin $B_{9}, 6.8-36.3$ $\mathrm{nmol} / \mathrm{l}$; vitamin $B_{12}, 200-900 \mathrm{pg} / \mathrm{ml}$; vitamin $B_{1}$, 50-150 nmol/l; vitamin C, 34-114 $\mu \mathrm{mol} / \mathrm{l}$; vitamin $B_{2}, 4.26-18.42 \mu \mathrm{g} / \mathrm{l}$; and vitamin $B_{6}, 14.6-72.9$ $\mathrm{nmol} / \mathrm{l}$.

\section{Chemotherapy regimens}

Of these 178 patients, 135 received induction chemotherapy, 11 patients received postoperative adjuvant chemotherapy, and 32 received retreatment. All the drugs were administered intravenously and repeated every 3 weeks. The specific regimens were as follows: 1) for SCLC patients: etoposide and platinum; and irinotecan and platinum, and 2) for NSCLC patients: taxanes or pemetrexed and platinum.

\section{Ethical approval}

Ethical approval was given by the IRB of Chinese PLA General Hospital with the following reference number: S2019-198-02.

\section{Statistical analysis}

Statistical analysis was performed using the SPSS 24.0 software package. Non-normally distributed measurement data are presented with quartiles and medians, and the inter-group com- parisons were based on rank sum test. Count data were compared using the $\chi^{2}$ test. The potential correlation between vitamin concentrations and body mass index (BMI) before and after chemotherapy was analysed. A $p$-value $<0.05$ was considered significantly different.

\section{Results}

\section{Changes in BMI and haematological indicators in patients with lung cancer before and after chemotherapy}

BMI decreased in 101 (56.74\%) patients after chemotherapy. The number of anaemia patients increased from 96 before chemotherapy to 145 after chemotherapy. The proportions of patients with hypoproteinaemia or electrolyte disorders showed no significant change after chemotherapy (Table II).

Nutritional status of vitamins in patients with lung cancer before and after chemotherapy

Vitamin A, D, C, and $B_{2}$ deficiencies were observed during chemotherapy. After chemotherapy, the proportions of vitamin $\mathrm{D}, \mathrm{C}$, and $\mathrm{B}_{2}$ deficiencies increased significantly (Table III). No deficiency or excess of vitamins $B_{1}, B_{6}, B_{9}$, or $B_{12}$ was observed in all 178 patients.

Table I. Baseline data of lung cancer patients $(n=178)$

\begin{tabular}{|c|c|c|}
\hline Clinical features & $\begin{array}{l}\text { Number of } \\
\text { patients }\end{array}$ & $\begin{array}{c}\text { Percentage } \\
(\%)\end{array}$ \\
\hline \multicolumn{3}{|l|}{ Gender: } \\
\hline Males & 138 & 77.53 \\
\hline Females & 40 & 22.47 \\
\hline \multicolumn{3}{|l|}{ Age [years]: } \\
\hline$<60$ & 77 & 48.73 \\
\hline$\geq 60$ & 101 & 56.74 \\
\hline \multicolumn{3}{|l|}{ Clinical stage: } \\
\hline Stage III & 80 & 44.94 \\
\hline Stage IV & 98 & 55.06 \\
\hline \multicolumn{3}{|l|}{ Pathologic type: } \\
\hline Small-cell lung cancer & 74 & 41.57 \\
\hline Adenocarcinoma & 58 & 32.58 \\
\hline Squamous cell carcinoma & 46 & 25.84 \\
\hline \multicolumn{3}{|l|}{ Treatment modalities: } \\
\hline Induction chemotherapy & 135 & 75.84 \\
\hline $\begin{array}{l}\text { Postoperative adjuvant } \\
\text { chemotherapy }\end{array}$ & 11 & 6.18 \\
\hline $\begin{array}{l}\text { Chemotherapy after } \\
\text { recurrence }\end{array}$ & 32 & 17.98 \\
\hline
\end{tabular}


Table II. Changes in BMI and haematological indicators in 178 patients with lung cancer before and after chemotherapy, $n$ (\%)

\begin{tabular}{|c|c|c|}
\hline Item & $\begin{array}{c}\text { Before } \\
\text { chemotherapy }\end{array}$ & $\begin{array}{c}\text { After } \\
\text { chemotherapy }\end{array}$ \\
\hline \multicolumn{3}{|l|}{ BMI $\left[\mathrm{kg} / \mathrm{m}^{2}\right]:$} \\
\hline$<18.5$ & $4(2.25)$ & $5(2.81)$ \\
\hline $18.5-23.9$ & $83(46.63)$ & $84(47.19)$ \\
\hline$>23.9$ & $91(51.12)$ & $89(50.00)$ \\
\hline \multicolumn{3}{|l|}{ Haemoglobin: } \\
\hline Decreased & $96(53.92)$ & $145(81.46)$ \\
\hline Normal & $82(46.07)$ & 33 (18.54) \\
\hline \multicolumn{3}{|c|}{ Serum albumin: } \\
\hline Decreased & $31(17.42)$ & 32 (17.98) \\
\hline Normal & $147(82.58)$ & $146(82.02)$ \\
\hline \multicolumn{3}{|c|}{ Blood potassium: } \\
\hline Decreased & $12(6.74)$ & $21(11.78)$ \\
\hline Normal & $166(93.26)$ & $157(88.20)$ \\
\hline \multicolumn{3}{|l|}{ Blood sodium: } \\
\hline Decreased & $7(3.93)$ & $16(8.99)$ \\
\hline Normal & $171(96.07)$ & $162(91.01)$ \\
\hline \multicolumn{3}{|c|}{ Blood calcium: } \\
\hline Decreased & $28(15.73)$ & $22(12.36)$ \\
\hline Normal & $150(84.27)$ & $156(87.64)$ \\
\hline \multicolumn{3}{|c|}{ Blood phosphorus: } \\
\hline Decreased & $28(15.73)$ & $24(13.48)$ \\
\hline Normal & $150(84.27)$ & $154(86.51)$ \\
\hline
\end{tabular}

Comparison of vitamin concentrations and $\mathrm{BMI}$ in lung cancer patients before and after chemotherapy

There were statistically significant differences in vitamin $D, C$, and $B_{2}$ concentrations as well as $\mathrm{BMI}$ in 178 patients before and after chemotherapy (all $p<0.05)$ (Table IV).
Univariate analysis of vitamin levels in lung cancer patients before chemotherapy

Before chemotherapy, the concentrations of vitamin A and vitamin C were significantly different between males and females and among patients in different clinical stages (both $p<0.05$ ), the concentrations of vitamin $C$ and vitamin $B_{2}$ significantly differed among different pathological types of lung cancer $(p<0.05)$, and vitamin D level was significantly related to the disease status $(p<0.05)$ (Table V).

\section{Comparison of vitamin deficiencies in patients with different pathological types of lung cancer before chemotherapy}

The proportion of vitamin $B_{2}$ deficiency was significantly different among different pathological types $(p<0.05)$. However, the proportions of vitamins $A, D$, and $C$ deficiencies was not significantly correlated with the pathological types (all $p>0.05$ ) (Table VI).

\section{Comparison of the differences of vitamin concentrations and BMI in lung cancer patients during chemotherapy}

There was a correlation between the D-value of vitamin B1 concentration and the D-value of BMI before and after chemotherapy in 178 patients $(p<0.05)$ (Table VII).

\section{Discussion}

In the present study, 178 patients with lung cancer were tested for 9 vitamins. The results showed that the proportion of vitamin $B_{2}$ deficiency before and after chemotherapy was the highest, accounting for $48.31 \%$ and $56.18 \%$, respectively. No deficiency or excess of vitamins $B_{1}, B_{6}, B_{9}$, and $B_{12}$ was observed. The proportion of vitamin $B_{2}$ deficiency in lung cancer patients increased significantly after two cycles of chemotherapy, which may be related to the decrease in food intake or the imbalance of dietary structure during chemotherapy. Further analysis revealed that the change in serum vitamin B1 concentration before and after chemotherapy in patients with lung

Table III. Nutritional status of vitamins in 178 patients with lung cancer before and after chemotherapy

\begin{tabular}{|lcccc|}
\hline Vitamin & \multicolumn{2}{c|}{ Before chemotherapy } & \multicolumn{2}{c|}{ After chemotherapy } \\
\cline { 2 - 5 } & Normal & Deficient & Normal & Deficient \\
\hline A & $135(75.84)$ & $43(24.16)$ & $157(88.20)$ & $21(11.80)$ \\
\hline D & $166(93.26)$ & $12(6.74)$ & $135(75.84)$ & $43(24.16)$ \\
\hline C & $111(62.36)$ & $67(37.64)$ & $105(58.99)$ & $73(41.01)$ \\
\hline$B_{2}$ & $92(52.69)$ & $86(48.31)$ & $78(43.82)$ & $100(56.18)$ \\
\hline
\end{tabular}


Table IV. Comparison of vitamin concentrations and BMI in 178 lung cancer patients before and after chemotherapy

\begin{tabular}{|lcccc|}
\hline Vitamin & $\begin{array}{c}\text { Before chemotherapy (Q1-Q3) } \\
\text { median }\end{array}$ & $\begin{array}{c}\text { After chemotherapy (Q1-Q3) } \\
\text { median }\end{array}$ & $Z$ & $P$-value \\
\hline A & $(0.522-0.946) 0.718$ & $(0.498-0.871) 0.637$ & -1.723 & 0.085 \\
\hline D & $(32.181-47.430) 38.537$ & $(28.981-44.053) 35.273$ & -3.719 & 0.000 \\
\hline E & $(10.778-11.509) 11.076$ & $(10.824-11.645) 11.197$ & -1.188 & 0.235 \\
\hline$B_{9}$ & $(15.405-24.683) 19.859$ & $(15.433-23.785) 19.562$ & -1.766 & 0.077 \\
\hline$B_{12}$ & $(475.091-599.012) 536.719$ & $(478.295-598.756) 542.221$ & -0.101 & 0.920 \\
\hline B1 & $(77.658-97.014) 85.445$ & $(78.338-98.496) 85.230$ & -0.616 & 0.538 \\
\hline C & $(34.070-40.774) 36.432$ & $(33.103-37.951) 34.894$ & -3.807 & 0.000 \\
\hline$B_{2}$ & $(4.049-4.915) 4.270$ & $(4.001-4.619) 4.184$ & -2.209 & 0.027 \\
\hline$B_{6}$ & $(29.776-36.783) 32.218$ & $(29.757-35.594) 31.790$ & -0.891 & 0.373 \\
\hline BMl & $(22.200-26.125) 24.050$ & $(21.875-26.025) 23.950$ & -2.474 & 0.013 \\
\hline
\end{tabular}

Q1 and Q3, quartiles.

Table V. Univariate analysis of vitamin levels in lung cancer patients before chemotherapy

\begin{tabular}{|lcccccccccc|}
\hline Vitamin & $\begin{array}{c}\text { Age } \\
(<60 / \geq 60 \text { years })\end{array}$ & \multicolumn{2}{c}{$\begin{array}{c}\text { Gender } \\
\text { (males/females) }\end{array}$} & $\begin{array}{c}\text { Clinical stage } \\
\text { (III/IV) }\end{array}$ & \multicolumn{2}{c|}{$\begin{array}{c}\text { Disease status } \\
\text { (treatment-naive/ } \\
\text { recurrent) }\end{array}$} & $\begin{array}{c}\text { Pathologic type } \\
\text { (SCLC/adenocarci- } \\
\text { noma/SCC) }\end{array}$ \\
\cline { 2 - 11 } & $Z$ & $P$-value & $Z$ & $P$-value & $Z$ & $P$-value & $Z$ & $P$-value & $Z$ & $P$-value \\
\hline A & -1.565 & 0.118 & -3.894 & 0.000 & -1.971 & 0.049 & -0.307 & 0.759 & 2.305 & 0.316 \\
\hline D & -0.427 & 0.669 & -0.673 & 0.501 & -0.907 & 0.365 & -2.386 & 0.017 & 1.585 & 0.453 \\
\hline C & -0.733 & 0.464 & -2.060 & 0.039 & -2.377 & 0.017 & -1.078 & 0.281 & 6.133 & 0.047 \\
\hline$B_{2}$ & -0.266 & 0.790 & -0.045 & 0.964 & -0.721 & 0.471 & -0.614 & 0.539 & 6.145 & 0.046 \\
\hline
\end{tabular}

The descriptive statistics of vitamin concentrations before chemotherapy are shown in Table IV. SCLC - small cell lung cancer, SCC - squamous cell cancer.

Table VI. Comparison of vitamin deficiencies in patients with different pathological types of lung cancer before chemotherapy, $n(\%)$

\begin{tabular}{|lcccccccc|}
\hline \multirow{2}{*}{ Vitamin } & \multicolumn{2}{c}{ Small-cell lung cancer } & \multicolumn{2}{c}{ Adenocarcinoma } & \multicolumn{2}{c}{ Squamous cell cancer } & \multirow{2}{*}{$\chi^{2}$} & \multirow{2}{*}{$P$-value } \\
\cline { 2 - 7 } & Normal & Deficient & Normal & Deficient & Normal & Deficient & & \\
\hline A & $59(79.73)$ & $15(20.27)$ & $42(72.41)$ & $16(27.59)$ & $34(73.91)$ & $12(26.09)$ & 1.076 & 0.584 \\
\hline D & $66(89.19)$ & $8(10.81)$ & $56(96.55)$ & $2(3.45)$ & $44(95.65)$ & $2(4.35)$ & 2.899 & 0.236 \\
\hline C & $50(67.57)$ & $24(32.43)$ & $35(60.34)$ & $23(39.66)$ & $26(56.52)$ & $20(43.48)$ & 1.623 & 0.444 \\
\hline$B_{2}$ & $42(56.76)$ & $32(43.24)$ & $34(58.62)$ & $24(41.38)$ & $16(34.78)$ & $30(65.22)$ & 7.142 & 0.028 \\
\hline
\end{tabular}

cancer was correlated with the changes in BMI. $B$ vitamins are actually a group of water-soluble vitamins including $B_{9}$ (folate), $B_{6}$ (pyridoxine), $B_{2}$ (riboflavin), and $B_{1}$ (thiamine). Many studies have shown that serum vitamin $\mathrm{B}_{6}$ level is negatively correlated with the risk of lung squamous cell carcinoma, especially in males and individuals with a history of smoking [20, 21]. However, it has also been reported that the risk of lung cancer is not associated with vitamin $\mathrm{B}_{6}$ intake, and the additional vitamin $B_{6}$ supplementation in non-lung cancer population may even increase the risk of lung cancer [22]. Vitamin $\mathrm{B}_{2}$, also known as riboflavin, is mainly derived from foods such as meat, dairy products, soy products, and fresh fruits and vegetables [23]. Studies have shown that riboflavin is an antioxidant nutrient that can prevent lipid peroxidation and reperfusion injury. Riboflavin deficiency may increase the risk of oesophageal cancer, gastric cancer, colorectal cancer, and other malignant tumours [24-26]. In 2019, Chantarawong et al. [27] found that lumichrome, a major 
Table VII. Relationship between the changes of vitamin concentrations and BMI before and after chemotherapy

\begin{tabular}{|lcc|}
\hline D-values of vitamin & \multicolumn{2}{c|}{ D-values of BMI } \\
\cline { 2 - 3 } concentrations & $r$ & $P$-value \\
\hline Vitamin A & 0.097 & 0.196 \\
\hline Vitamin D & -0.083 & 0.271 \\
\hline Vitamin $\mathrm{E}$ & 0.004 & 0.955 \\
\hline Vitamin $\mathrm{B}_{9}$ & 0.025 & 0.742 \\
\hline Vitamin $\mathrm{B}_{12}$ & 0.085 & 0.257 \\
\hline Vitamin $\mathrm{B}_{1}$ & 0.171 & 0.023 \\
\hline Vitamin $\mathrm{C}$ & -0.030 & 0.686 \\
\hline Vitamin $\mathrm{B}_{2}$ & -0.107 & 0.154 \\
\hline Vitamin $\mathrm{B}_{6}$ & 0.122 & 0.104 \\
\hline
\end{tabular}

derivative of riboflavin, might exhibit pharmacological activity against cancer cells. It could inhibit the growth of lung cancer cells and reduce their survival rate. Their study might help the research and development of this compound for the treatment of lung cancer.

We found that patients with lung cancer had varying degrees of vitamin $A$ and $D$ deficiencies. There was a correlation between vitamin A level and clinical stage before chemotherapy, and the vitamin A concentration was significantly lower in patients with stage IV lung cancer than in those with stage III lung cancer. The vitamin D level was correlated with disease status in lung cancer patients before chemotherapy. The vitamin D level was significantly lower in patients with relapsed lung cancer than in treatment-naive patients. After chemotherapy, the vitamin D concentration decreased in all patients, and the proportion of vitamin D deficiency increased compared with that before chemotherapy. Chemotherapy drugs and diet may affect serum vitamin $A$ and $D$ levels in patients with lung cancer, and they are also associated with disease progression. Whether vitamin $D$ deficiency affects the prognosis of lung cancer patients needs further investigation. Both vitamin $A$ and vitamin D are fat-soluble vitamins. Because they dissolve in fat and are not easily excreted, they can be stored in the body tissues. Daily supplies are not required for these two vitamins. Studies have found that vitamin D is an anti-tumour nutrient that can promote cell apoptosis, reduce tumour proliferation, and increase the tumour cells' sensitivity to chemotherapeutic drugs; it may also be associated with the decreased risk of lung cancer, colon cancer, breast cancer, and other tumours $[28,29]$. The results of a randomised double-blind controlled trial suggested that vita- min D supplementation in patients with NSCLC could increase the survival rate of patients with early lung adenocarcinoma [30]. Yu et al. [31] concluded in a meta-analysis that increased intake of vitamin A and carotene could reduce the risk of lung cancer, especially in Asian populations; unfortunately, all the relevant studies were limited by the lack of dose-response analysis.

In the present study, the number of lung cancer patients with vitamin C deficiency was 67 (37.64\%) before chemotherapy, which increased to $73(41.01 \%)$ after chemotherapy, especially in females and patients with stage IV lung cancer. Further analysis of the vitamin C deficiency in patients with different pathological types of lung cancer showed that the proportion of patients with squamous cell carcinoma was the largest, and the proportion of vitamin C deficiency reached $43.48 \%$. Thus, serum vitamin C may be a sensitive marker for lung cancer, and special attention should be paid to this vitamin during micronutrient supplementation. Vitamin $C$ is a water-soluble vitamin. It acts as an antioxidant at low concentrations but a pro-oxidant at high concentrations. Vitamin C can inhibit glycolysis of tumour cells, arrest tumour cell cycle, and induce tumour cell apoptosis, thus playing an anti-tumour role [32]. A case-control study of lung cancer in Canada found that antioxidants such as vitamin $C$ and carotene could lower the risk of lung cancer (including squamous cell carcinoma, adenocarcinoma, and small cell carcinoma) in female moderate smokers and might also be helpful for male heavy smokers [33]. In a randomised controlled study, Ou et al. [34] investigated the value of vitamin $\mathrm{C}$ combined with chemotherapy and supportive treatment in 97 patients with refractory advanced NSCLC and found that combined vitamin C and chemotherapy could increase the treatment efficacy and improve the quality of life. However, we did not offer vitamin C supplementation in our patients. Whether vitamin treatment affects the efficacy and prognosis of patients warrants further investigations in controlled studies with larger sample sizes.

To further investigate the correlation between vitamin levels and the efficacy and adverse reactions of chemotherapy in lung cancer patients, we analysed 135 newly diagnosed patients with lung cancer who did not undergo surgery. Seventy-one (52.59\%), 44 (32.59\%), and 20 (14.81\%) patients showed partial remission (PR), stable disease (SD), and progressive disease (PD), respectively, after two cycles of chemotherapy, and the clinical benefit rate $(P R+S D)$ reached $85.19 \%$. At the same time, 135 patients were divided into a normal vitamin group and a vitamin-deficiency group, according to the vitamin level before chemotherapy, and statistical analysis was performed in terms of efficacy, bone marrow suppression, and electrolyte 
disorder in the two groups. There was no significant difference between the two groups $(p>0.05)$.

Subgroup analysis showed that the efficacy and side effects of chemotherapy in patients with non-small-cell lung cancer and small cell lung cancer did not differ significantly among different vitamin groups (all $p>0.05$ ).

This was an observational study in which no additional vitamin supplementation was given to patients with vitamin-deficient lung cancer, and nutritional interventions were conducted in patients with malnutrition during chemotherapy in accordance with the 5-step treatment principle.

By analysing the changes of vitamin concentration in lung cancer patients before and after chemotherapy, we found that chemotherapy had a certain impact on the vitamin nutritional status of patients, while the chemotherapy tolerance was attenuated in patients with severe vitamin deficiency. The efficacy of chemotherapy and prognosis in lung cancer patients are associated with a variety of factors. Vitamins may be involved in the occurrence and development of lung cancer. However, according to the findings in our current study, it cannot be inferred that vitamin level has a direct correlation with the efficacy and adverse reactions of chemotherapy. Whether vitamin supplementation can improve the effect of chemotherapy or the prognosis of lung cancer patients remains inconclusive.

Subsequently, we will design a prospective interventional study in order to further explore the impact of vitamin nutritional status on the efficacy and prognosis of lung cancer patients.

Several limitations of the study deserve mention. First, it was a retrospective study, and the sample size is relatively small. Second, there was no group with supplementation of vitamins to make a comparison. Third, the modes of chemotherapy applied varied, which might have a confounding effect. Moreover, in our study, we did not have data about overall survival and response to treatment, which is of great importance because no randomised trial has conclusively demonstrated improved survival as result of vitamin supplementation during chemotherapy. Randomised prospective studies are needed to better define the optimal supplementation of vitamins and their impact on survival and side effects of chemotherapy in patients with lung cancer undergoing CT. For these reasons, we need to validate our findings in a multicentre prospective study in the future.

In conclusion, vitamin deficiency is common in patients undergoing chemotherapy for lung cancer, among which vitamin $B_{2}, C, D$, and A deficiencies are more frequent. Chemotherapy has a certain effect on the changes in vitamin concentrations in lung cancer patients. Nutrition- al assessment and vitamin determination are important for patients with lung cancer during chemotherapy. Standard nutritional therapy and appropriate vitamin supplementation can help improve the nutritional status of patients, reduce chemotherapy complications, and improve treatment tolerance and compliance.

\section{Acknowledgments}

This research was supported by:Project Funds on the Health Bureau of the Logistics support Department of the Central military Commission (17BJZ47). Lanqing Liang, Wei Yu, Boning Cai contributed equally to this work.

\section{Conflict of interest}

The authors declare no conflict of interest.

\section{References}

1. National Lung Screening Trial Research Team. Lung cancer incidence and mortality with extended follow-up in the national lung screening trial. J Thorac Oncol 2019; 14: $1732-42$.

2. Zhou G. Tobacco, air pollution, environmental carcinogenesis, and thoughts on conquering strategies of lung cancer. Cancer Biol Med 2019; 16: 700-13.

3. Bray F, Ferlay J, Soerjomataram I, Siegel RL, Torre LA, Jemal A. Global cancer statistics 2018: GLOBOCAN estimates of incidence and mortality worldwide for 36 cancers in 185 countries. CA Cancer J Clin 2018; 68: 394-424.

4. Xu R, Han Y. Long non-coding RNA FOXF1 adjacent non-coding developmental regulatory RNA inhibits growth and chemotherapy resistance in non-small cell lung cancer. Arch Med Sci 2019; 15: 1539-46.

5. Huang N, Dai W, Li Y, Sun J, Ma C, Li W. LncRNA PCAT-1 upregulates RAP1A through modulating miR324-5 $p$ and promotes survival in lung cancer. Arch Med Sci 2019; 16: 1196-206.

6. Wang X, Arcani DMC, Zhao J, Xu M, Zhou X, Yang Y. Prognostic and diagnostic significance of Cavin 2 in lung adenocarcinoma. Arch Med Sci 2019; 16: 1189-95.

7. Seyfried TN. Cancer as a mitochondrial metabolic disease. Front Cell Dev Biol 2015; 3: 43.

8. Kumar S, Lombard DB. Mitochondrial sirtuins and their relationships with metabolic disease and cancer. Antioxid Redox Signal 2015; 22: 1060-77.

9. Nasir A, Bullo MMH, Ahmed Z, et al. Nutrigenomics: epigenetics and cancer prevention: a comprehensive review. Crit Rev Food Sci Nutr 2020; 60: 1375-87.

10. Jain A, Tiwari A, Verma A, Jain SK. Vitamins for cancer prevention and treatment: an insight. Curr Mol Med 2017; 17: 321-40

11. Álvaro Sanz E, Garrido Siles M, Rey Fernández L, et al. Nutritional risk and malnutrition rates at diagnosis of cancer in patients treated in outpatient settings: early intervention protocol. Nutrition 2019; 57: 148-53.

12. Song C, Cao J, Zhang F, et al. Nutritional risk assessment by scored patient-generated subjective global assessment associated with demographic characteristics in 23,904 common malignant tumors patients. Nutr Cancer 2019; 71: 50-60. 
13. Arends J, Baracos V, Bertz $\mathrm{H}$, et al. ESPEN expert group recommendations for action against cancer-related malnutrition. Clin Nutr 2017; 36: 1187-96.

14. Arends J, Bachmann P, Baracos V, et al. ESPEN guidelines on nutrition in cancer patients. Clin Nutr 2017; 36: 11-48.

15. Irimie Al, Braicu C, Pasca S, et al. Role of key micronutrients from nutrigenetic and nutrigenomic perspectives in cancer prevention. Medicina 2019; 55: 283.

16. Gröber U, Holzhauer P, Kisters K, Holick MF, Adamietz IA. Micronutrients in oncological intervention. Nutrients 2016; 8: 163.

17. Nasir A, Bullo MMH, Ahmed Z, et al. Nutrigenomics: epigenetics and cancer prevention: a comprehensive review. Crit Rev Food Sci Nutr 2020; 60: 1375-87.

18. Zhang XS, Liu Z, Peng Y, et al. Analysis of vitamin nutritional status in hospitalized cancer patients. Chin J Clin Oncol Rehabil 2018; 25: 1448-51.

19. Hoffer LJ, Robitaille L, Swinton N, et al. Appropriate vitamin $D$ loading regimen for patients with advanced lung cancer. Nutr J 2016; 15: 84.

20. Fanidi A, Muller DC, Yuan JM, et al. Circulating folate, vitamin B6, and methionine in relation to lung cancer risk in the Lung Cancer Cohort Consortium (LC3). J Natl Cancer Inst 2018; 110: 57-67.

21. Theofylaktopoulou D, Midttun $\varnothing$, Ueland PM, et al. Impaired functional vitamin B6 status is associated with increased risk of lung cancer. Int J Cancer 2018; 142: 2425-34

22. Brasky TM, White E, Chen CL. Long-term, supplemental, one-carbon metabolism-related vitamin b use in relation to lung cancer risk in the vitamins and lifestyle (VITAL) cohort. J Clin Oncol 2017; 35: 3440-8.

23. Saedisomeolia A, Ashoori M. Riboflavin in human health: a review of current evidences. Adv Food Nutr Res 2018; 83: 57-81.

24. Tutino V, Defrancesco ML, Tolomeo M, et al. The expression of riboflavin transporters in human colorectal cancer. Anticancer Res 2018; 38: 2659-67.

25. Thakur K, Tomar SK, Singh AK, Mandal S, Arora S. Riboflavin and health: a review of recent human research. Crit Rev Food Sci Nutr 2017; 57: 3650-60.

26. Pan F, Luo HJ, Wu ZY, et al. Decreased plasma riboflavin is associated with poor prognosis, invasion, and metastasis in esophageal squamous cell carcinoma. Eur J Clin Nutr 2020; 74: 1149-56.

27. Chantarawong W, Kuncharoen N, Tanasupawat S, Chanvorachote $P$. Lumichrome inhibits human lung cancer cell growth and induces apoptosis via a p53-dependent mechanism. Nutr Cancer 2019; 71: 1390-402.

28. Vojdeman FJ, Madsen CM, Frederiksen K, et al. Vitamin D levels and cancer incidence in 217,244 individuals from primary health care in Denmark. Int J Cancer 2019; 145: 338-46.

29. Maalmi H, Ordóñez-Mena JM, Schöttker B, Brenner $H$. Serum 25-hydroxyvitamin D levels and survival in colorectal and breast cancer patients: systematic review and meta-analysis of prospective cohort studies. Eur J Cancer 2014; 50: 1510-21.

30. Akiba T, Morikawa T, Odaka M, et al. Vitamin D supplementation and survival of patients with non-small cell lung cancer: a randomized, double-blind, placebo-controlled trial. Clin Cancer Res 2018; 24: 4089-97.

31. Yu N, Su X, Wang Z, Dai B, Kang J. Association of dietary vitamin a and beta-carotene intake with the risk of lung cancer: a meta-analysis of 19 publications. Nutrients 2015; 7: 9309-24
32. Roa FJ, Pena E, Gatica M, et al. Therapeutic use of vitamin C in cancer: physiological considerations. Front Pharmacol 2020; 11: 211.

33. Shareck M, Rousseau MC, Koushik A, Siemiatycki J, Parent ME. Inverse association between dietary intake of selected carotenoids and vitamin c and risk of lung cancer. Front Oncol 2017; 7: 23

34. Ou J, Zhu X, Chen P, et al. A randomized phase II trial of best supportive care with or without hyperthermia and vitamin C for heavily pretreated, advanced, refractory non-small-cell lung cancer. J Adv Res 2020; 24: 175-82. 